\title{
HIV care coverage among HIV-positive adolescent girls and young women in South Africa: Results from the HERStory Study
}

C Mathews, ${ }^{1,2 *} \mathrm{PhD} ;$ M Cheyip, ${ }^{3 *} \mathrm{MSc}$ (Med); R Beauclair, ${ }^{4} \mathrm{PhD} ;$ A Puren, ${ }^{5,6} \mathrm{MB}$ ChB, $\mathrm{PhD} ;$ C Lombard, ${ }^{7,8} \mathrm{PhD} ;$ K Jonas, ${ }^{1,2} \mathrm{PhD}$; K A Ayalew, ${ }^{3}$ MPhil; D Govindasamy, ${ }^{1,2} \mathrm{PhD}$; C Kuo, ${ }^{9,10}$ DPhil, MPhil; J Dietrich, ${ }^{11} \mathrm{PhD}$; F Abdullah, ${ }^{12,13} \mathrm{MB}$ ChB, FCPHM; G Gray, ${ }^{14} \mathrm{MB}$ ChB, FC Paed (SA)

${ }^{1}$ Health Systems Research Unit, South African Medical Research Council, Cape Town, South Africa

${ }^{2}$ Adolescent Health Research Unit, Division of Child and Adolescent Psychiatry, Department of Psychiatry and Mental Health, Faculty of Health Sciences, University of Cape Town, South Africa

${ }^{3}$ Centers for Disease Control and Prevention, Pretoria, South Africa

${ }^{4}$ South African Department of Science and Technology/National Research Foundation (DST-NRF) Centre of Excellence in Epidemiological Modelling and Analysis (SACEMA), Stellenbosch University, South Africa

${ }^{5}$ National Institute of Communicable Diseases, Johannesburg, South Africa

${ }^{6}$ Division of Virology, School of Pathology, Faculty of Health Sciences, University of the Witwatersrand, Johannesburg, South Africa ${ }^{7}$ Biostatistics Unit, South African Medical Research Council, Cape Town, South Africa

${ }^{8}$ Division of Epidemiology and Biostatistics, Department of Global Health, Faculty of Medicine and Health Sciences, Stellenbosch University, Cape Town, South Africa

${ }^{9}$ Brown University, Providence, RI, USA

${ }^{10}$ Department of Psychiatry and Mental Health, Faculty of Health Sciences, University of Cape Town, South Africa

${ }^{11}$ Perinatal HIV Research Unit (PHRU), School of Clinical Medicine, Faculty of Health Sciences, University of the Witwatersrand, Johannesburg, South Africa

${ }^{12}$ Office of AIDS and TB Research, South African Medical Research Council, Pretoria, South Africa

${ }^{13}$ Division of Infectious Diseases, Department of Medicine, School of Medicine, Faculty of Health Sciences, University of Pretoria and Steve Biko Academic Hospital, Pretoria, South Africa

${ }^{14}$ Office of the President, South African Medical Research Council, Cape Town, South Africa

* Joint first authors

Corresponding author: C Mathews (catherine.mathews@mrc.ac.za)

Background. Health service coverage cascades measure the proportion of a population in need of a service that experienced a positive health outcome from the service, and enable tracking of progress in achieving universal health coverage and inequities in care coverage.

Objectives. To investigate HIV care coverage among HIV-positive adolescent girls and young women (AGYW) living in six South African districts, compare coverage by age and socioeconomic status (SES), and investigate other associated factors including participation in a combination HIV prevention intervention.

Methods. The HERStory Study was an evaluation of the combination intervention, comprising a representative household survey of AGYW aged 15 - 24 years living in six intervention districts. From September 2017 to November 2018, biological, sociodemographic and behavioural data were collected. HIV-positive status, initiation of antiretroviral therapy (ART) and viral suppression were determined through laboratory tests (enzyme-linked immunosorbent assay for HIV antibodies, antiretroviral (ARV) metabolites and viral load (VL) testing). Viral suppression was defined as a VL $<1000$ copies $/ \mathrm{mL}$. Knowledge of HIV-positive status was self-reported, and participants testing positive for ARV metabolites were assumed to have known their HIV-positive status. Unconditional HIV care cascades were created, stratified by age and SES. We used Pearson's $\chi^{2}$ tests corrected for survey-based analysis to describe factors associated with knowledge of HIV status, and being on ART.

Results. Of the 4399 participants, 568 were HIV-positive (12.4\%), of whom 60.8\% (95\% confidence interval (CI) 57.1 - 64.5) knew their status, $50.6 \%$ (95\% CI 46.6 - 54.0) were on ART, and 62.1\% (95\% CI 58.4 - 65.9) were virally suppressed. Most participants (84.9\%) were in the lower SES group, and they had better coverage than the higher SES group: 61.9\% (95\% CI 58.3 - 65.4) knew their status, 52.1\% (95\% CI 48.4 - 55.9) were on ART, and 64.9\% (95\% CI 61.3 - 68.4) were virally suppressed, compared with 55.0\% (95\% CI 42.1 - 68.0), 40.0\% (95\% CI 29.2 - 50.8), and $46.6 \%$ (95\% CI 34.5 - 58.7), respectively. Participants aged 15 - 19 years had slightly inferior coverage to the 20 - 24-year-old group: $57.5 \%$ knew their status, $46.1 \%$ were on ART and $59.5 \%$ were virally suppressed, compared with $62.3 \%, 52.2 \%$ and $63.3 \%$.

Conclusions. These findings emphasise the need to close the gaps in HIV care coverage among AGYW, of whom only $61 \%$ knew their HIVpositive status and only $62 \%$ were virally suppressed. There is pro-poor inequality in HIV care coverage, with those in lower socioeconomic groups more likely to be virally suppressed.

S Afr Med J 2021;111(5):460-468. https://doi.org/10.7196/SAMJ.2021.v111i5.15351 
Health interventions during adolescence and young adulthood have the potential to generate a triple dividend: improved health during adolescence and young adulthood, during later adulthood, and for the next generation. ${ }^{[1,2]}$ For adolescents (aged $10-19$ years) and young people (aged 20 - 24 years) living with HIV, who are among the most vulnerable populations, ${ }^{[3]}$ HIV diagnosis and treatment interventions have the potential to reduce mortality, improve health and wellbeing, and halt transmission to other individuals. However, adolescents and young people have had inadequate access to HIV diagnosis and treatment, which contributes to avoidable AIDSrelated morbidity and deaths. ${ }^{[4]}$ For example, compared with adults on antiretroviral therapy (ART), adolescents diagnosed with HIV have poorer adherence to ART ${ }^{[5]}$ and are the only age group with increasing HIV mortality. ${ }^{[6]}$

South Africa (SA) has the world's largest ART programme, which has increased life expectancy and saved lives, ${ }^{[7]}$ as well as cut maternal-to-child transmission (MTCT) of HIV from 25 - 30\% before 2001 to $1.4 \%$ in $2016{ }^{[8]}$ However, SA adolescents and young people are less likely than adults to benefit from HIV interventions, and are less likely to be diagnosed and to know their HIV positive status compared with older people ${ }^{[9]}$ many with HIV do not start $\mathrm{ART}^{,[10]}$ and more than half of HIV-positive young men and women aged 15 - 24 years are not virally suppressed. ${ }^{[9]}$ Compared with adult mothers, adolescent mothers living with HIV are more likely to have unplanned pregnancies and less likely to access interventions to prevent MTCT. ${ }^{[1]}$

Combination HIV prevention and care interventions, which merge effective biomedical, behavioural and structural interventions for combined delivery, are one of the key strategies for reaching the 90-9090 targets and achieving the Sustainable Development Goal (SDG) of ending the HIV epidemic by 2030. ${ }^{[12]}$ Between 2016 and 2019, the Global Fund made an investment of USD67 million in a 3-year comprehensive combination HIV prevention and care programme for SA adolescent girls and young women (AGYW) aged $10-24$ years. The intervention was implemented by government and non-government organisations in 10 districts in which young women were at high risk of HIV acquisition and in which there was no other large-scale HIV prevention intervention. In each district, the intervention was targeted to selected subdistricts, areas or wards where it was determined that risk of HIV was highest. Key intervention components were Soul Buddyz Clubs ${ }^{[13]}$ for adolescent girls and boys aged $10-14$ years in primary schools, the Keeping Girls in School (KGS) programme for AGYW aged $14-18$ years in high schools (https://www.mietafrica. $\mathrm{com} /$ projects-programmes/youth-development/keeping-girls-inschool-kgs/), RISE Clubs for AGYW in school aged 15 - 19 years, and RISE Clubs and Women of Worth Clubs for AGYW out of school aged 19 - 24 years. HIV testing and linkage to HIV prevention and care services were offered through the Club and KGS programmes. HIV testing was also promoted indirectly, by referring AGYW to clinics. In some districts, young people were employed as clinic 'navigators' and placed in clinics to meet and welcome AGYW and to promote youthresponsive clinic services.

Global initiatives such as the United Nations SDGs, the Lancet Commission on Adolescent Health and Wellbeing ${ }^{[1,2]}$ and Countdown to 2030 have called attention to tracking progress in the health of adolescents and young people up to age 24 years. The concept of 'effective coverage' (EC), defined as the proportion of a population in need of a service that experienced a positive health outcome from the service, is critical to measuring such progress. ${ }^{[14]}$ Health service coverage cascades have been proposed as the most appropriate way to measure EC, and enable us to monitor progress in achieving universal health coverage and a high-quality health system. ${ }^{[14,15]}$ They enable the measurement of inequities in care coverage: as HIV care coverage increases, there may be uneven progress in reaching subpopulations, and inequities may remain or even increase. ${ }^{[16]}$ It is important to monitor disparities in access to care by socioeconomic status (SES). Socioeconomic inequalities are associated with inequities in sexual and reproductive health (SRH) among adolescents. For example, in sub-Saharan Africa, poorer adolescent girls (compared with wealthier) face more barriers in meeting their SRH needs and are more likely to have their first sexual encounter and to start childbearing at a younger age. ${ }^{[17]}$

\section{Objectives}

We used HIV care coverage cascades stratified by age and SES to describe the coverage of HIV care services among AGYW aged 15 - 24 years living in geographical areas in which the combination intervention was implemented. We also investigated whether age, SES (poverty level), being in high school, having a deceased parent, recency of HIV infection, and participation in key components of the combination intervention were associated with care coverage.

\section{Methods}

\section{Study design and sampling}

The data were generated from the HERStory Study, an evaluation of the combination intervention. For these analyses, the design was a cross-sectional, representative household survey of AGYW aged 15 - 24 years living in six of the 10 districts in which the combination HIV prevention intervention was implemented: City of Cape Town (Western Cape Province), Ehlanzeni (Mpumalanga Province), OR Tambo (Eastern Cape Province), Tshwane (Gauteng Province), King Cetshwayo and Zululand (KwaZulu-Natal Province). The survey began in 2017, 18 months after the start of the intervention, and it was completed in 2018, 32 months after implementation started.

We used a stratified cluster sampling design, with three stages of sampling. First, we took a simple random sample of census, small area layers (SALs) within the sub-areas in which the intervention was implemented in each district. Then we conducted a systematic random sample of $35 \%$ of households within each SAL. Finally, all AGYW aged 15 - 24 years in each household were invited to participate in the study. If the members of a selected household declined to participate, we did not replace the household. Our original sample size calculation of 14000 AGYW, in 10 districts (Table 1), was based on being able to measure a difference in HIV incidence over 2 years using cross-sectional data. ${ }^{[18]}$ However, the survey could only be completed in six districts within the time allocated to the study, and the four districts scheduled last for data collection were not completed.

\section{Measures}

We used electronic questionnaires developed using the Mobenzi Researcher data collection software suite (https://www.mobenzi. com/researcher/home), administered using a tablet. Demographic, socioeconomic and behavioural data were collected from all enrolled participants using these structured electronic questionnaires administered by trained fieldworkers.

The questionnaire included a number of categorical variables related to SES: ${ }^{[19]}$ (i) AGYW was away from home for more than 1 month in the past 12 months (internal migration has been shown to cause and be caused by poverty; ${ }^{[20]}$ (ii) has piped water in household; (iii) has a flushing toilet in household; (iv) household has working electricity; $(v)$ household has a car; $(v i)$ household has a computer; (vii) household has internet; (viii) household has 
a refrigerator; (ix) household has a stove; (x) AGYW or household member went a day/night without eating in the past month; (xi) AGYW has own money; (xii) AGYW saves money; and (xiii) AGYW owes money.

We asked about participation in the key components of the combination HIV prevention intervention, which were branded and therefore easy to identify. A participant was defined as having participated if she reported ever attending or being a member of Soul Buddyz or RISE or Women of Worth, or had ever attended a KGS health education or homework support session.

The HIV status of participants was determined using blood samples that were analysed in a laboratory. The samples were tested with the Bio-Rad HIV1/2 Combo Assay (Genscreen, France) and any reactive result was confirmed by a second 4th-generation test (HIV1/2 COMBI COBAS E411 (Roche Diagnostics, Germany). All positive specimens were confirmed for HIV-1 infection by Western blot (GS HIV-1 Western Blot, Bio-Rad Laboratories, USA).

To distinguish recent HIV infection, we used a limiting antigen avidity immunoassay (LAg EIA; Maxim Biomedical, USA). Specimens confirmed by Western blot to be HIV-positive were tested to determine recent or early v. long-term HIV infection using the single-well LAg-Avidity EIA test (Maxim Biomedical). Recent HIV infections had a mean duration of 161 days. The HIV-1 RNA viral load (VL) assay and ART drug measurements were included in a recent infection testing algorithm to minimise the false recent rate, to determine the proportion of HIV-infected persons on ART and those who were ART naive with detectable and undetectable VLs.

Participants were asked whether they had ever had an HIV test, and if yes, what their test result was at the most recent test. Later in the questionnaire, they were asked whether they knew their HIV status (negative, positive, unknown). Participants were classified as having knowledge of their HIV-positive status if they answered that they were positive in either of those two questions. In addition, they were assumed to have knowledge of their status if a laboratory test confirmed that they were positive and antiretroviral (ARV) metabolites were present in their blood.

ART testing was performed on HIV serology-positive specimens for measurement of ARVs that were in use in either first- or second-line regimens in the public sector. Antiretroviral testing was performed using dry blood spots (DBSs) determined by high-performance liquid chromatography (HPLC) coupled with tandem mass spectrometry (HPLC-Module 1260 Infinity 11 (Agilent Technologies Inc., Germany), mass spectrometer ABSciex 6.5+ (USA). The assay was a validated qualitative detection of nevirapine, emtricitabine, lamuvidine, abacavir and tenofovir (the lower limit of detection was $25 \mathrm{ng} / \mathrm{mL} / 0.025 \mu \mathrm{g} / \mathrm{mL}$ ), efavirenz, and lopinavir (lower limit of detection $100 \mathrm{ng} / \mathrm{mL} / 0.1 \mu \mathrm{g} / \mathrm{mL})$. Known standards were analysed with every batch of samples to ensure reproducibility and adequate quality assurance. If any metabolite was detected in their blood, the participant was considered to have ART exposure.

To determine VL, HIV-1 VL testing was performed on all confirmed HIV-positive specimens using the Abbott m2000 HIV Real-Time System (Abbott Molecular Inc., USA). Viral suppression was defined as a cut-off $\leq 1000$ copies $/ \mathrm{mL}$.

\section{Procedures}

The field team identified the sampled households using aerial maps, and determined the geographical co-ordinates using the Global Positioning System (GPS). If the selected household was vacant, there was no AGYW in the household, or the household head declined to complete a household listing form to determine whether there were eligible AGYW, the household on the right was visited and assessed for eligibility. Only one eligible household was enrolled at the random point selected through this approach. We obtained consent from AGYW, and parental consent for AGYW $<18$ years of age. Trained fieldworkers first administered the survey to consenting AGYW. The sections of the questionnaire with questions about sexuality, HIV testing and HIV status were completed by the participants themselves to diminish social desirability bias. The fieldworker read each question to the participant and allowed the participant to enter her responses in the tablet privately. Then the fieldworker collected two microtainers of whole blood using a finger prick. After the questionnaire had been completed and specimens collected, participants were offered rapid HIV testing in the household. Microtainers of blood were shipped daily to the laboratory for preparation of DBSs and centrifugation to obtain plasma. Specimens not shipped on the same day were stored at $4-8^{\circ} \mathrm{C}$ until shipped the next day. Participants were reimbursed with a gift and voucher to the value of ZAR75 (USD5) to compensate them for their time. They were invited to 
visit their nearest clinic 2 weeks after their participation to obtain the results of the study laboratory tests, using a bar-coded referral card. The study was approved by the South African Medical Research Council Research Ethics Committee (ref. no. EC03611/2016) and by the Center for Global Health Associate Director for Science, US Centers for Disease Control and Prevention (CDC) (ref. no. CGH 2017-194a).

\section{Analysis}

The analyses were restricted to participants who tested HIV-positive in the study laboratory tests $(n=568)$, except for a description of the HIV prevalence in the study population. Since the aim was to generalise study results to the broader population of AGYW across all six districts and interpret the estimates as true population-level estimates, we incorporated sample weights into the analysis, and all estimates are weighted. The sample weights take into account the probability of sampling SALs in each district and the systematic probability of sampling households within each SAL. Survey-based analysis was performed with the six districts specified as survey strata, and SALs as the primary sampling unit. Finite population sampling estimation was used in the survey analysis to improve the precision of the estimates, and the number of SALs in each district was used for this approach.

We produced overall and stratified unconditional HIV care cascades for the HIV-positive AGYW in the study. The cascades presented here summarise: (i) the proportion of HIV-positive AGYW who know their status ('status known'); (ii) the proportion of HIVpositive AGYW who had ARV metabolites detected in their blood ('on ART'); and (iii) the fraction of HIV-positive AGYW who were virally suppressed ('virally suppressed'). The HIV care cascades are 'unconditional' because each proportion across the cascade uses the number of HIV-positive AGYW in the denominator.

Stratification variables included age group (15 - 19/20 - 24 years) and SES (relatively low/relatively high). A participant's SES group was determined using cluster analysis with the K-modes algorithm, ${ }^{[21]}$ with the 13 SES questions described above. Cluster analysis is an exploratory and unsupervised machine learning technique that allows analysts to divide data into meaningful groups based upon shared features. For further details about the SES variable, see Appendix A (available as a supplementary file at http://samj.org.za/ public/sup/15351.pdf).

HIV-positive participants were characterised by calculating descriptive statistics for the overall population and by knowledge of their HIV status. We also described the participants who were HIVpositive and knew their status, by ART status. For these bivariate analyses, Pearson's $\chi^{2}$ test was used, corrected for the survey-based analysis to describe whether age group, SES group, orphanhood (one or both parents deceased), being in school, recency of HIV infection, and self-reported participation in the AGYW combination intervention were associated with knowledge of HIV status, and being on ART. Risk differences and their 95\% confidence intervals (CIs) were also calculated for each of these bivariate analyses.

Stata 15.1 (StataCorp, USA) and R version 3.5.0 (R Core Team, Austria) were used to perform the analyses. ${ }^{[22]} \mathrm{In} \mathrm{R}$ the 'survey' and 'srvyr' packages were used for the survey-based analyses. ${ }^{[23,24]}$ The package 'klaR' was used for the cluster analysis. ${ }^{[25]}$

\section{Results \\ Sampling realisation and response rates of participants}

The data were collected during 2017 - 2018. In the six districts, we sampled 7300 AGYW and visited 22388 households. All the primary sampling units were visited in each of the districts (Table 1). The number of households visited met the target, but the number of ineligible households was higher in the urban-based districts, which led to a lower sample realisation. The overall sample realisation of participants was $60.6 \%$ in the six completed districts. Ultimately, 4436 AGYW completed the survey, and 4399 were in the age range, were enrolled in the study and were weighted to the total sample size of 7300 .

Table 2. Characteristics of the sample ( $N=568)$ of HIV-positive adolescent girls and young women aged 15 - 24 years in six districts in South Africa, $2017-2018^{*}$

\begin{tabular}{lll}
\hline Characteristics & $\boldsymbol{n}(\mathbf{\%})$ & $\mathbf{9 5 \%} \mathbf{C I}$ \\
\hline Age group (years) & & $27.9-34.1$ \\
$\quad 15-19$ & $385(30.9)$ & $65.9-72.1$ \\
$\quad 20-24$ & & $61.3-67.9$ \\
Currently in school & $354(64.6)$ & $32.1-38.7$ \\
$\quad$ No & $214(35.4)$ & \\
$\quad$ Yes & & $35.9-42.6$ \\
Has a deceased parent ${ }^{\dagger}$ & $219(39.2)$ & $57.4-64.1$ \\
$\quad$ No & $345(60.8)$ & \\
$\quad$ Yes & & $81.1-88.2$ \\
Socioeconomic status & $490(84.9)$ & $11.8-18.9$ \\
$\quad$ Relatively low socioeconomic group & $78(15.1)$ & \\
$\quad$ Relatively high socioeconomic group & & $53.1-60.4$ \\
Self-reported exposure to a Global Fund intervention & $334(56.8)$ & $39.6-46.9$ \\
$\quad$ No & $234(43.2)$ & \\
$\quad$ Yes & & $94.0-96.7$ \\
Recent HIV infection & $542(95.5)$ & $3.3-6.0$
\end{tabular}




\section{Characteristics of participants}

Of the 4399 participants in the broader study, 568 were HIV-positive (12.4\%) and comprised the study population for the analyses reported here. Most of the HIVpositive participants were in the 20 - 24-year age range $(69.1 \%), 35.4 \%$ were enrolled in high school, $84.9 \%$ fell into the relatively low SES group, and $43.2 \%$ reported they had participated in a key component of the combination HIV prevention intervention (Table 2). We classified $4.5 \%$ of participants as having recently been infected with HIV.

\section{Overall HIV care coverage}

Of all 568 participants with laboratoryconfirmed HIV-positive status, $60.8 \%$ (95\% CI 57.1 - 64.5) knew their status, $50.3 \%$ of 568 (95\% CI 46.6 - 54.0) were on ART, and $62.1 \%$ of 568 (95\% CI 58.4 - 65.9) were virally suppressed (Fig. 1). Interestingly, there was a higher fraction of AGYW who were virally suppressed, than of AGYW who knew their status.

\section{Age-stratified HIV care coverage}

A slightly smaller fraction of participants in the younger age group knew their HIV status (57.5\%; 95\% CI 52.1 - 62.8) compared with the older age group (62.3\%; 95\% CI 57.6 67.0) (Fig. 2). Only $46.1 \%$ (95\% CI 40.5 51.7) of younger HIV-positive AGYW were on ART compared with 52.2 (95\% CI 47.7 - 56.7) of older HIV-positive AGYW. Following a similar pattern, 59.5\% (95\% CI 54.1 - 64.8) of AGYW aged 15 - 19 years were virally suppressed, while $63.3 \%$ (95\% CI 58.4 - 68.2) of AGYW aged 20 - 24 years were virally suppressed.

\section{SES-stratified HIV care coverage}

In Fig. 3, the HIV care cascades, disaggregated by level of SES, show that participants in the lower SES group had better HIV care cascades: $61.9 \%$ (95\% CI 58.3 - 65.4) knew their status, $52.1 \%$ (95\% CI 48.4 - 55.9) were on ART, and 64.9\% (95\% CI 61.3 - 68.4) were virally suppressed, compared with $55.0 \%$ (95\% CI 42.1 - 68.0), 40.0\% (95\% CI 29.2 50.8 ) and $46.6 \%$ (95\% CI 34.5 - 58.7), respectively, in the relatively high SES group. The differences were most pronounced for viral suppression, indicating higher levels of care coverage for the relatively low SES group.

\section{HIV care coverage stratified by age and SES}

Participants in the older age and relatively high SES group had the worst cascades (Fig. 4). Approximately half in this group did not know their status, and nearly two-

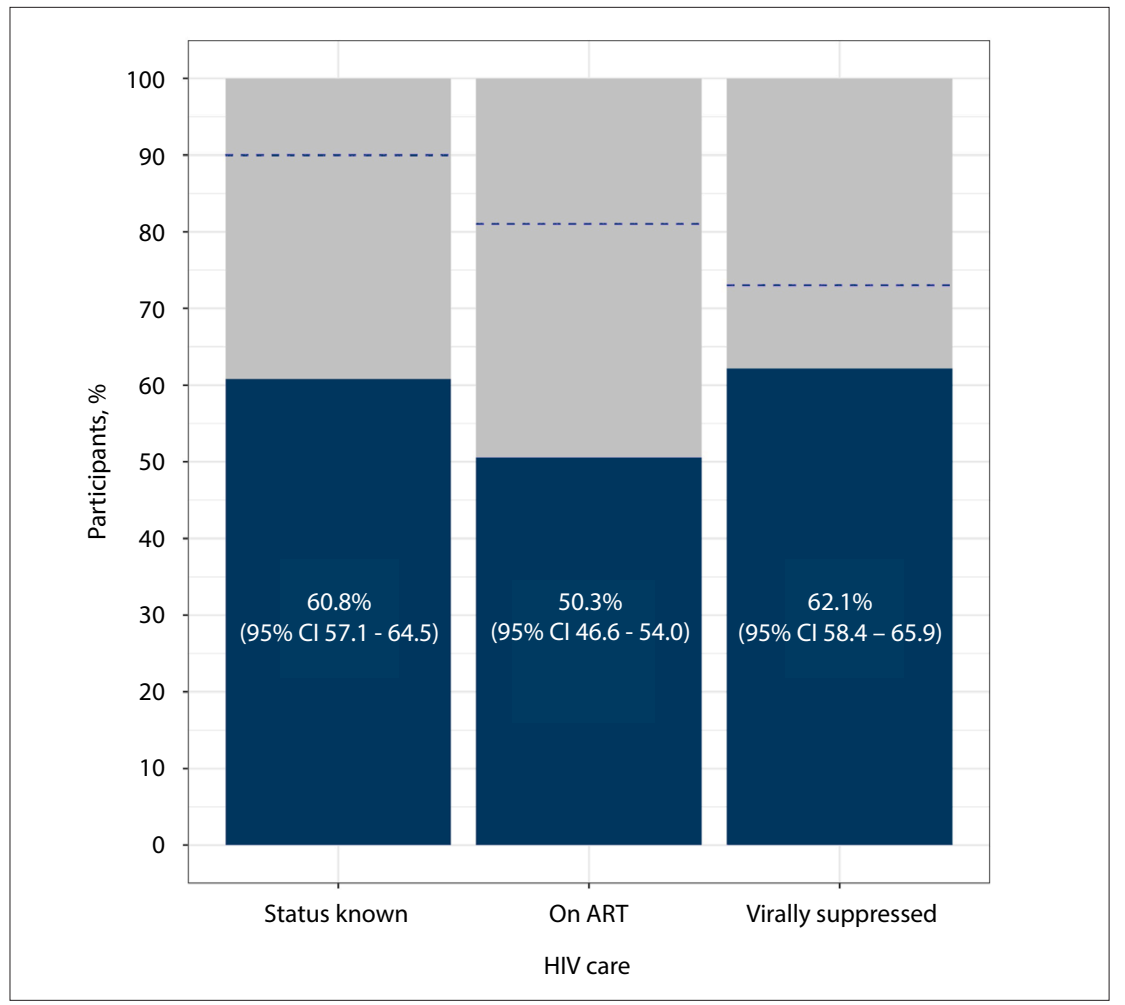

Fig. 1. HIV care coverage among 568 adolescent girls and young women aged $15-24$ years in six districts in South Africa, 2017 - 2018. (CI = confidence interval; $A R T=$ antiretroviral therapy.)

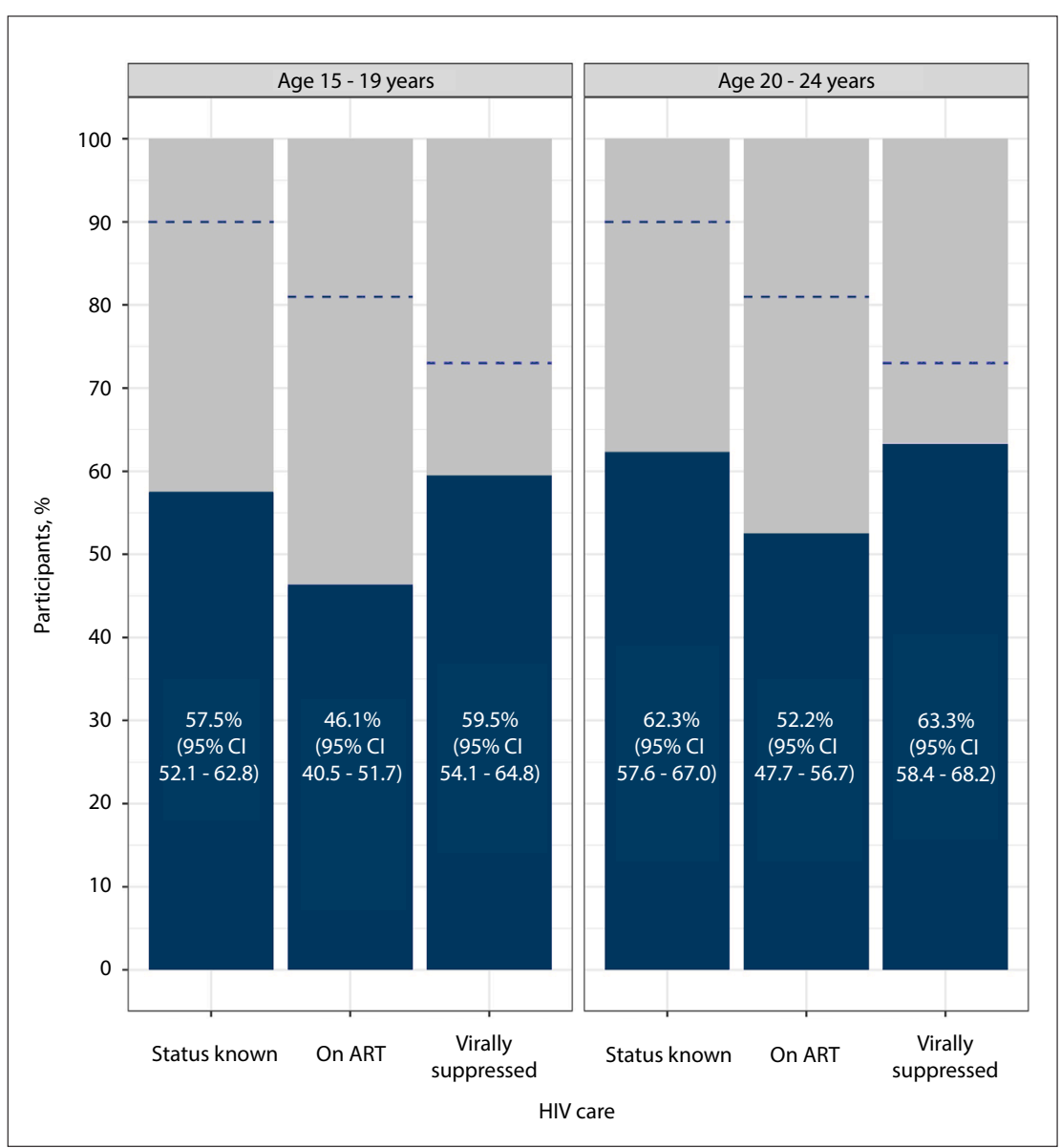

Fig. 2. HIV care coverage among 568 adolescent girls and young women aged $15-24$ years in six districts in South Africa, 2017 - 2018, stratified by age group. ( $C I=$ confidence interval; $A R T=$ antiretroviral therapy.) 


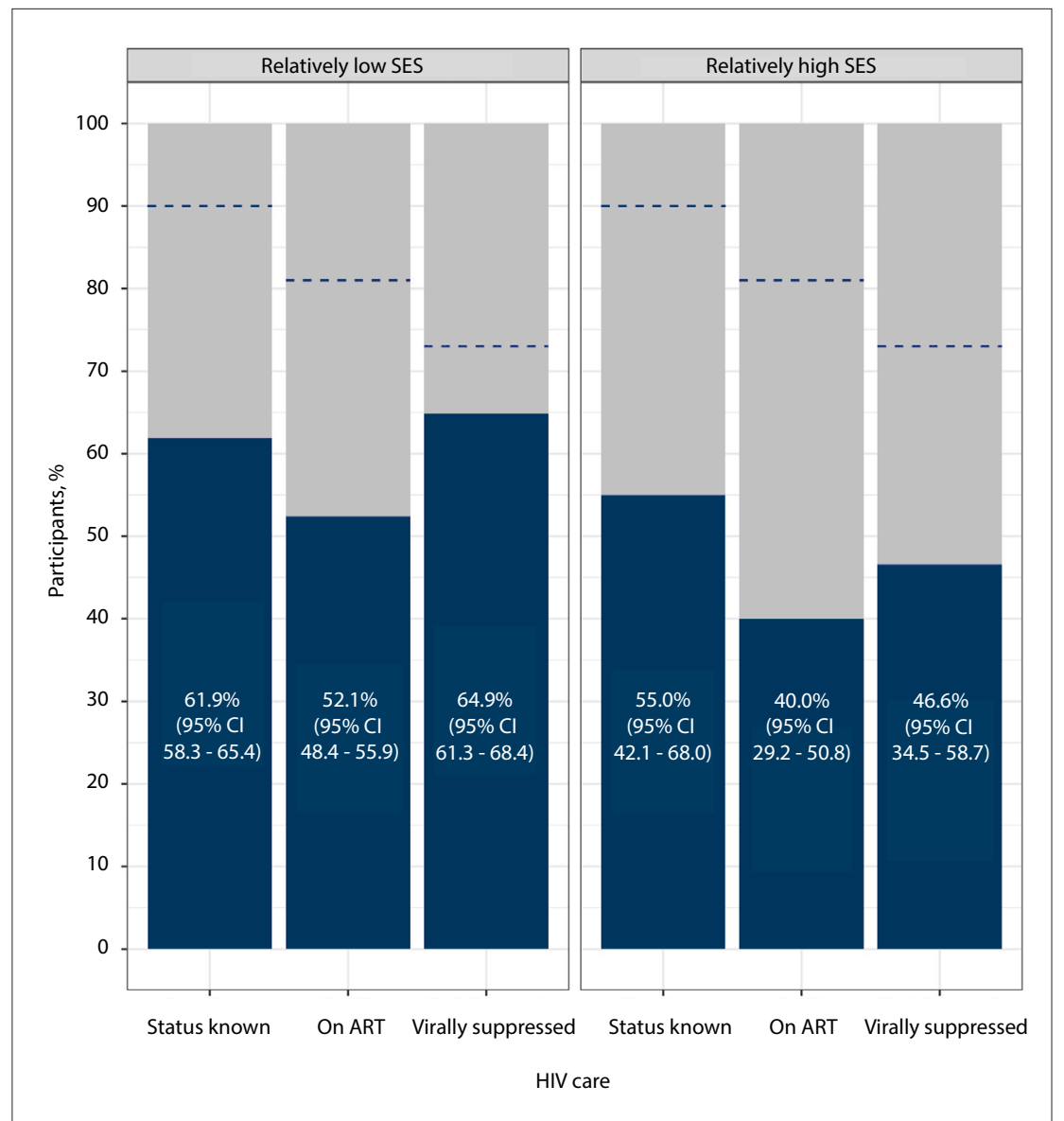

Fig. 3. HIV care coverage among 568 adolescent girls and young women aged $15-24$ years in six districts in South Africa, 2017 - 2018, stratified by SES. (SES = socioeconomic status; CI = confidence interval; $A R T=$ antiretroviral therapy.)

thirds were not on treatment. Those in the older age group who were in the relatively low SES group had the best outcomes, with $64.4 \%$ knowing their status, $55.4 \%$ being on treatment and $66.5 \%$ being virally suppressed.

\section{Factors associated with HIV care coverage}

Table 3 examines differences between participants who knew about their HIV-positive status v. those who did not. Among HIVpositive AGYW, 54.5\% (95\% CI 49.0 - 59.9) who did not have a deceased parent knew their status v. $64.5 \%$ (95\% CI 59.2 - 69.5) of those with a deceased parent, with a risk difference of $10.0 \%$ (95\% CI 2.8 - 17.2). Those who had recently been infected with HIV had 55.2 fewer instances of knowing their status per 100 AGYW (95\% CI -64.1 -46.2), compared with those with long-term infections $(63.3 \%$ v. $8.2 \%$; $p<0.000)$.

To develop hypotheses about why people who knew their HIV-positive status were not on ART, a description of these participants is presented in Table 4. Overall, 358 participants were HIV-positive and knew to be HIV-positive than children who are not orphaned. ${ }^{[26]}$ It is likely that young people who have lost a parent to HIV have had their own HIV diagnosed through the process of their parent's diagnosis or death.

To achieve the Joint United Nations Programme on HIV and AIDS (UNAIDS) target for the third ' 90 ', we need to achieve viral suppression in $73 \%$ of the population of HIV-positive AGYW. We have demonstrated a gap in HIV care coverage, with only $62.1 \%$ of the AGYW in our study virally suppressed. Nevertheless, the level of care coverage among AGYW in our study population, which was receiving an HIV combination prevention intervention, is substantially higher than the estimates from a nationally representative survey conducted in 2017, which found that only $47.7 \%$ of all HIV-positive AGYW aged 15 - 24 years were virally suppressed ${ }^{[9]}$ The substantially higher estimates in our study raise the question about whether the combination HIV prevention intervention, which was being implemented at the time of the survey, may have been contributing to closing gaps in HIV care coverage. Supporting this, we found that a higher fraction of participants on ART had participated in the combination HIV intervention compared with those not on ART. However, our study design does not enable us to draw conclusive evidence about the intervention effect.

Adolescents (aged 15-19 years) had slightly poorer levels of care coverage than young women aged $\geq 20$ years of age. This is consistent with other studies that show the difficulty of achieving EC of HIV care among adolescents in SA and sub-Saharan Africa. ${ }^{[4-6,27]}$ We found that AGYW in the lower SES group had substantially better levels of care coverage than those in the higher SES group. A similar pro-poor inequality in care coverage has been observed in the uptake of HIV testing among pregnant women in SA. ${ }^{[28]}$ A possible explanation is that AGYW who were employed were more likely to be in the higher SES group and had little time away from work to access HIV services. Another possible explanation is that the free HIV care services provided by the public sector were more acceptable to AGYW in the lower SES group compared with those in the higher SES group, while at the same time private sector HIV care services were not accessible to either group. The disparities in levels of care coverage by SES group suggest that efforts to make services more youth-friendly need to consider the accessibility, acceptability and appropriateness of the service for different AGYW subgroups. 


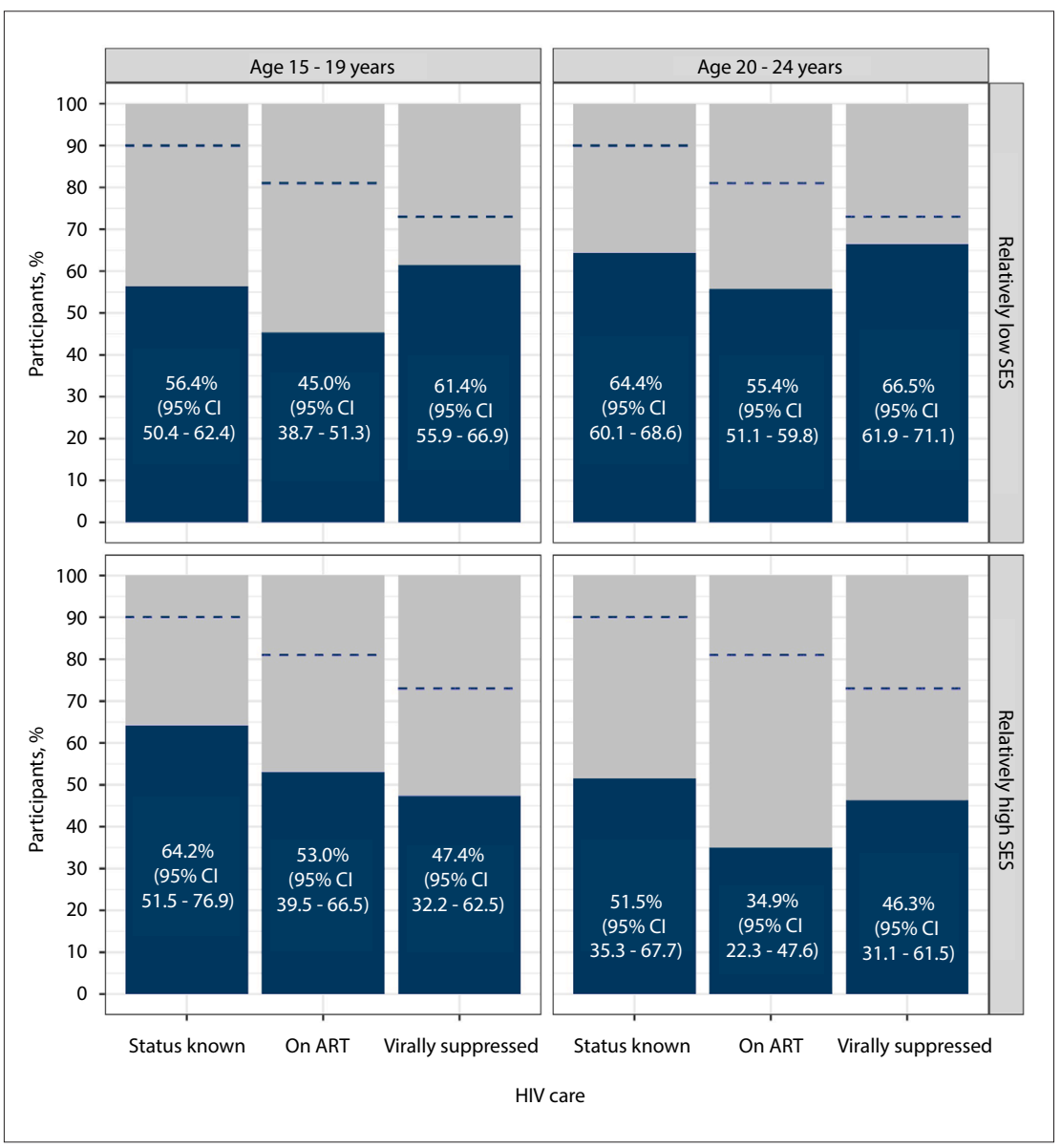

Fig. 4. HIV care coverage among 568 adolescent girls and young women aged $15-24$ years in six districts in South Africa, 2017 - 2018, stratified by age group and SES. (SES = socioeconomic status; $C I=$ confidence interval; $A R T=$ antiretroviral therapy.)

\section{Study limitations}

Some participants were virally suppressed but did not know their HIV status, based on our measures of knowledge of HIV status. Furthermore, some participants were determined to be virally suppressed, but ARVs were not detected in their blood, a phenomenon observed elsewhere. ${ }^{[9]}$ There are several possible explanations for these observations, including imperfect sensitivity of laboratory tests to detect ART. Another possible reason is that the progression of HIV in the absence of ART includes periods of time when the VL is lower than the threshold used for determining viral suppression. ${ }^{[29]}$ Furthermore, AGYW may be treated with ART by parents/guardians and not know their HIV status.

The cross-sectional study design limits our ability to attribute the higher levels of care coverage observed in the study population (compared with the national average) to the HIV prevention intervention that was being implemented. Furthermore, the survey was conducted during the 2 nd and 3 rd years of the intervention, and the intervention may not have had time to demonstrate impact on care coverage. The validity of participants' reports of participation in the intervention in unknown. The sample realisation of $61 \%$ is a limitation, but it compares well with the 2016 South African Demographic and Health Survey response rate $(56 \%$ among 15 - 19-year-olds and 57\% among 20 24-year-old women selected to provide samples for HIV testing). ${ }^{[30]}$

\section{Conclusions}

These findings emphasise weaknesses in HIV care for AGYW in these study districts, of whom 39\% did not know their HIVpositive status and would not have access to treatment. To achieve the UNAIDS target for the third ' 90 ' (viral suppression among 90\% of those who know their status and are on ART), viral suppression needs to be achieved in $73 \%$ of the population of HIV-positive AGYW. This study has demonstrated a gap in care coverage, with only $62.1 \%$ of the AGYW study population virally suppressed. Special efforts are needed to improve care coverage for adolescent girls and AGYW in the higher SES group, who have relatively low levels of HIV care coverage.

Declaration. None.

Acknowledgements. We acknowledge and thank the adolescent girls and young women and other participants who agreed to make themselves available to take part in this research, and share their views, opinions and experiences with us. The AGYW intervention was funded by the Global Fund to Fight AIDS, TB and Malaria (Global Fund). The combination HIV prevention interventions were implemented in the 10 districts by a range of government departments and civil society organisations that were appointed by the organisations responsible for the management of the AGYW programme: Western Cape Department of Health, KwaZuluNatal Treasury, Kheth'Impilo, Soul City Institute for Social Justice, and the Networking HIV and AIDS Community of Southern Africa. The programme was aligned with the She Conquers campaign and was implemented with support from the South African National AIDS Council through the Country Coordinating Mechanism (CCM) and the CCM Secretariat.

Author contributions. CM and MC conceptualised the study and wrote the manuscript. All authors were co-investigators on the study. RB and CL performed the statistical analysis. AP directed the laboratory methods. All authors reviewed and contributed to drafts of the manuscript.

Funding. This study has been supported by the US Department of Health and Human Services, CDC, Division of Global HIV \& TB, under the terms of Cooperative Agreement 1U2GGH001150. Development of this publication was supported by the Social Impact Bond of the South African Medical Research Council (SAMRC), funded by the Global Fund. The work herein for KJ and JD was made possible through funding by the SAMRC through its Division of Research Capacity Development under the intramural Post-doctoral Fellowship Programme from funding received from the South African National Treasury. JD was also funded in part through a CIPHER Growing the Leaders of Tomorrow grant from the International AIDS Society.

Conflicts of interest. None.

Disclaimer. The findings and conclusions in this article are those of the authors and do not necessarily represent the official position of the CDC. 


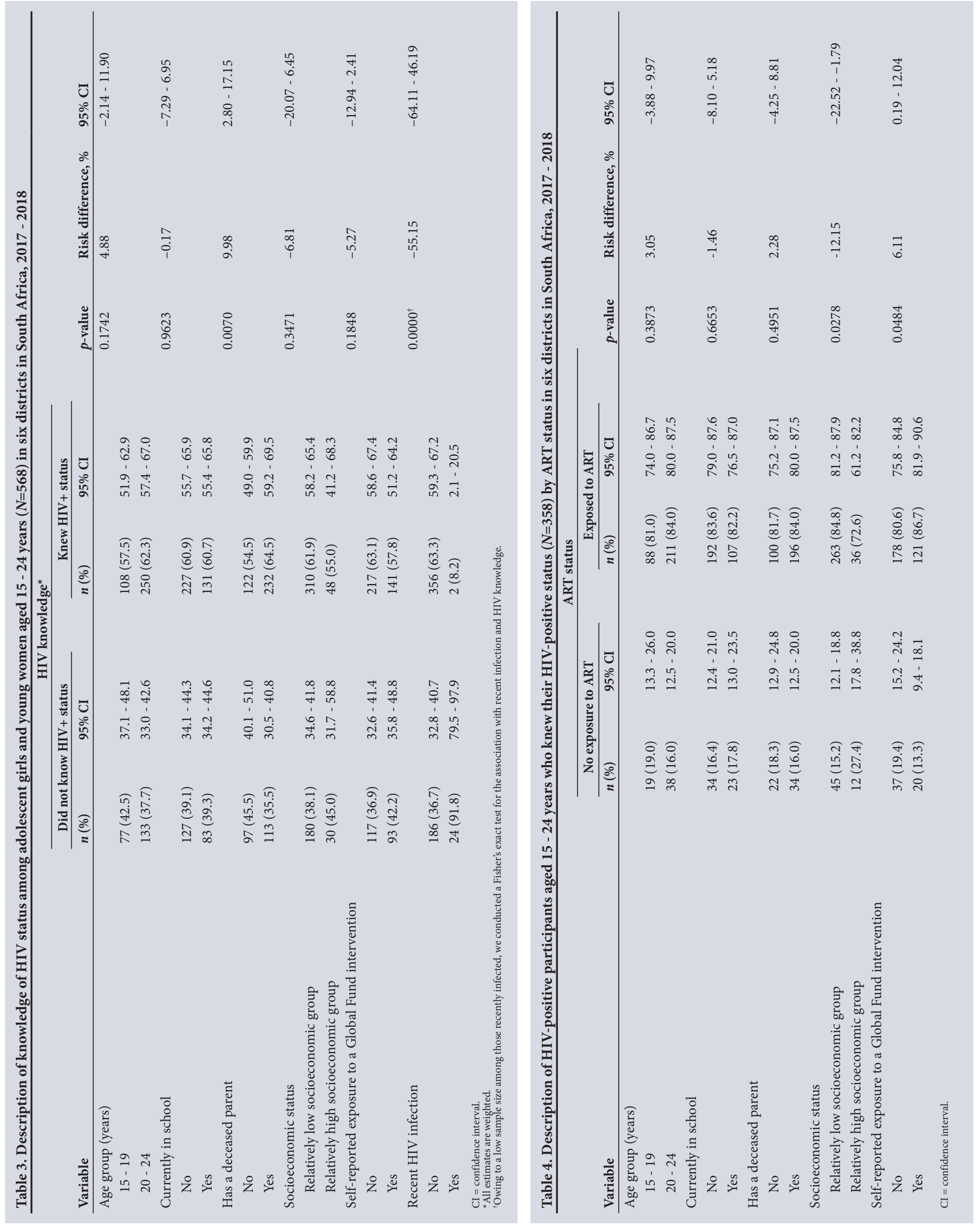


1. Azzopardi PS, Hearps SJC, Francis KL, et al. Progress in adolescent health and wellbeing: Tracking 12 headline indicators for 195 countries and territories, 1990 - 2016. Lancet 2019;393(10176):1101-1118. headline indicators for 195 countries and territor
https://doi.org/10.1016/S0140-6736(18)32427-9

2. Patton GC, Sawyer SM, Santelli JS, et al. Our future: A Lancet commission on adolescent health and . Patton GC, Sawyer SM, Santelli JS, et al. Our future: A Lancet commission on adolescent hea
wellbeing. Lancet 2016;387(10036):2423-2478. https://doi.org/10.1016/S0140-6736(16)00579-1

3. Nagata JM, Hathi S, Ferguson BJ, Hindin MJ, Yoshida S, Ross DA. Research priorities for adolescent health in low- and middle-income countries: A mixed-methods synthesis of two separate exercises J Glob Health 2018;8(1):010501. https://doi.org/10.7189/jogh.08.010501

4. Sam-Agudu NA, Folayan MO, Ezeanolue EE. Seeking wider access to HIV testing for adolescents in sub-Saharan Africa. Pediatr Res 2016;79(6):838-845. https://doi.org/10.1038/pr.2016.28

5. Adejumo OA, Malee KM, Ryscavage P, Hunter SJ, Taiwo BO. Contemporary issues on the epidemiology and antiretroviral adherence of HIV-infected adolescents in sub-Saharan Africa: A narrative review. J Int AIDS Soc 2015;18:20049. https://doi.org/10.7448/IAS.18.1.20049

6. Enane LA, Vreeman RC, Foster C. Retention and adherence: Global challenges for the long-term care of adolescents and young adults living with HIV. Curr Opin HIV AIDS 2018;13(3):212-219. https:// doi.org/10.1097/COH.0000000000000459

7. Dorrington R, Bradshaw D, Laubscher R, Nannan N. Rapid Mortality Surveillance Report 2017. Cape Town: South African Medical Research Council, 2019. https://www.samrc.ac.za/sites/default/files/ Town: South African Medical Research Council, 2019. https://wwW.samrc.ac.za/s

8. Goga A, Sherman G, Chirinda W, et al. Fliminating mother to child transmission of HIV in South Africa: 2002 - 2016, progress, challenges and the Last Mile Plan. In: South African Health Review. Durban: Health Systems Trust, 2017:137-146. http://www.hst.org.za/publications/south-africanhealth-review-2017 (accessed 1 April 2021)

9. Simbayi L. South African National HIV Prevalence, Incidence, Behaviour and Communication Survey, 2017. Boulder, Colo.: Lynne Rienner, 2019. https://www.hsrcpress.ac.za/books/south-african-nationahiv-prevalence-incidence-behaviour-and-communication-survey-2017 (accessed 1 April 2021).

10. Maskew M, Bor J, MacLeod W, Carmona S, Sherman GG, Fox MP. Adolescent HIV treatment in South Africa’s national HIV programme: A retrospective cohort study. Lancet HIV 2019;6(11):e760-e768. https://doi.org/10.1016/S2352-3018(19)30234-6

11. Ramraj T, Jackson D, Dinh TH, et al. Adolescent access to care and risk of early mother-to-child HIV transmission. J Adolesc Health 2018;62(4):434-443. https://doi.org/10.1016/j.jadohealth.2017.10.007

12. Joint United Nations Programme on HIV/AIDS (UNAIDS). 90-90-90: An ambitious treatment target to help end the AIDS epidemic. October 2014. https://www.unaids.org/sites/default/files/media asset/90-90-90_en.pdf (accessed 1 April 2021).

13. Johnson S, Magni S, Dube Z, Goldstein S. Extracurricular school-based social change communication program associated with reduced HIV infection among young women in South Africa. J Health Comm 2018;23(12):1044-1050. https://doi.org/10.1080/10810730.2018.1544675

14. Marsh AD, Muzigaba M, Diaz T, et al. Effective coverage measurement in maternal, newborn, child, and adolescent health and nutrition: Progress, future prospects, and implications for quality health systems. Lancet Glob Health 2020;8(5):e730-e736. https://doi.org/10.1016/S2214-109X(20)30104-2

15. Kruk ME, Gage AD, Arsenault C, et al. High-quality health systems in the Sustainable Development Goals era: Time for a revolution. Lancet Glob Health 2018;6(11):e1196-e1252. https://doi. org/10.1016/S2214-109X(18)30386-3
16. Assefa Y, Hill PS, Van Damme W, Dean J, Gilks CF. Leaving no one behind: Lessons from implementation of policies for universal HIV treatment to universal health coverage. Glob Health 2020;16(1):17. https://doi.org/10.1186/s12992-020-00549-4

17. Melesse DY, Mutua MK, Choudhury A, et al. Adolescent sexual and reproductive health in subSaharan Africa: Who is left behind? BMJ Glob Health 2020:5(1):e002231. https://doi.org/10.1136/ bmjgh-2019-002231

18. Konikoff J, Brookmeyer R. Sample size methods for estimating HIV incidence from cross-sectional surveys. Biometrics 2015;71(4):1121-1129. https:doi.org/10.1111/biom.12336

9. Rutstein SO, Johnson K. The DHS wealth index. DHS Comparative Reports No. 6. Calverton, Md ORC Macro, 2004. https://doi.org/10.13140/2.1.2806.4809

20. Crush J, Frayne B, eds. Surviving on the Move: Migration, Poverty and Development in Southern Africa. Pretoria: IDASA and Development Bank of Southern Africa, 2010.

21. Huang $\mathrm{Z}$. A fast clustering algorithm to cluster very large categorical data sets in data mining. In: $\mathrm{Lu} \mathrm{H}_{\mathrm{H}}$ Matoda H, Luu H, eds. KDD: Techniques and Applications. Singapore: World Scientific, 1997:21-34.

22. R Core Team: A language and environment for statistical computing. Vienna, Austria, 2018. https:// www.R-project.org/ (accessed 1 April 2021)

23. Lumley T. Analysis of complex survey samples. J Stat Softw 2019. https://doi.org/10.18637/jss.v009.i08

24. Freedman Ellis G. srvyr: 'dplyr'-like syntax for summary statistics of survey data. 2019. http://gdfe co/srvyr; https://github.com/gergness/srvyr (accessed 1 April 2021).

25. Weihs C, Ligges U, Luebke K, Raabe N. klaR analyzing German business cycles. In: Baier D, Decke R, Schmidt-Thieme L, eds. Data Analysis and Decision Support. Berlin: Springer-Verlag, 2005:334343. https://doi.org/10.1007/3-540-28397-8_36

26. Kidman R, Anglewicz P. Are adolescent orphans more likely to be HIV-positive? A pooled dat analyses across 19 countries in sub-Saharan Africa. J Epidemiol Comm Health 2016;70(8):791-797. https://doi.org/10.1136/jech-2015-206744

27. Green D, Tordoff DM, Kharono B, et al. Evidence of sociodemographic heterogeneity across the HIV treatment cascade and progress towards 90-90-90 in sub-Saharan Africa - a systematic review and meta-analysis. J Int AIDS Soc 2020;23(3):e25470. https://doi.org/10.1002/jia2.2547

28. Ngandu NK, Van Malderen C, Goga A, Speybroeck N. Wealth-related inequality in early uptake of HIV testing among pregnant women: An analysis of data from a national cross-sectional survey South Africa. BMJ Open 2017;7(7):e013362. https://doi.org/10.1136/bmjopen-2016-013362

29. Fraser C, Hollingsworth TD, Chapman R, de Wolf F, Hanage WP. Variation in HIV-1 setpoint viral load: Epidemiological analysis and an evolutionary hypothesis. Proc Nat Acad Sci 2007;104(44):17441-17446. https://doi.org/10.1073/pnas.0708559104

30. National Department of Health, South Africa, Statistics South Africa, South African Medical National Department of Health, South Africa, Statistics South Africa, South African Medica
Research Council, and ICF. South Africa Demographic and Health Survey 2016. Pretoria: NDoH Stats SA, SAMRC and ICF, 2019. http://www.samrc.ac.za/sites/default/files/attachments/2019-01-29/ SADHS2016.pdf (accessed 1 April 2021).

Accepted 17 November 2020. 